\title{
Visual Image Quality Assessment Technique using FSIM
}

\author{
Rohit Kumar \\ Csvtu bhilai \\ Sscet bhilai \\ India
}

\author{
Vishal Moyal \\ Csvtu bhilai \\ Sscet bhilai \\ India
}

\begin{abstract}
The goal of quality assessment (QA) research is to design algorithms that can automatically assess the quality of images in a perceptually consistent manner. Image QA algorithms generally interpret image quality as fidelity or similarity with a "reference" or "perfect" image in some perceptual space. In order to improve the assessment accuracy of white noise, Gauss blur, JPEG2000 compression and other distorted images, this paper puts forward an image quality assessment method based on phase congruency and gradient magnitude. The experimental results show that the image quality assessment method has a higher accuracy than traditional method and it can accurately reflect the image visual perception of the human eye. In this paper, we propose an image information measure that quantifies the information that is present in the reference image and how much of this reference information can be extracted from the distorted image.
\end{abstract}

Keywords: Image quality assessment (IQA), structural similarity index (SSIM), feature similarity index (FSIM), phase congruency (PC), gradient magnitude(GM), low level feature

\section{INTRODUCTION}

Image quality assessment is an important study topic in the image processing area. Image quality is a fundamental characteristic of any image which measures the perceived image degradation .Generally, compared with an ideal or perfect image. Digital images are subject to a wide variety of distortions during acquisition, processing, compression, storage, transmission and reproduction, any of which may result in a degradation of visual quality. Imaging systems introduces some amount of distortion or artifacts which reduces the quality assessment and here it is our point of interest.

By defining image quality in terms of a deviation from the ideal situation, quality measures become technical in the sense that they can be impartially determined in terms of deviations from the ideal models.

Generally speaking, visual quality assessment can be divided into two categories one is subjective visual quality assessment and another one is objective visual quality assessment.
Subjective quality assessment is done by humans which represents the realistic opinion towards an Image. Image quality objective assessment uses the mathematical model to quantitative the assessment index and simulates human visual perception system to assess the image quality. Common image quality objective assessment indexes include PSNR (Peak Signal to Noise Ratio), SSIM (Structural Similarity), and FSIM (Feature-Similarity). Based upon the Availability of Reference Objective quality assessment is classified as no reference (NR),reduced reference(RR),full reference(FR)[1]methods. If there is no reference signal available for the distorted (test) one to compare with, then a quality evaluation method is termed as a Noreference (NR).If the information of the reference medium is partially available, e.g., in the form of a set of extracted features, then this is the so-called Reduced-Reference (RR) method. FR method needs the complete reference medium to assess the distorted medium. Since it has the full information about original medium, 
it is expected to have the best quality prediction performance. Most existing quality assessment schemes belong to this category.

The following are implemented for image quality assessment algorithms as Mean square error (MSE), peak signal to noise (PSNR), structural similarity index (SSIM), feature similarity index (FSIM). Mean Squared Error is the average squared difference between a reference image and a distorted image. It is computed pixel-bypixel by adding up the squared differences of all the pixels and dividing by the total pixel count. Peak Signal-to-Noise Ratio is the ratio between the reference signal and the distortion signal in an image, given in decibels. The higher the PSNR, the closer the distorted image is to the original SNR(Peak signal to noise). The simplest and most widely used image quality metrics are MSE and PSNR since they are easy to calculate and are also mathematically convenient in the context of optimization. However, they often correlate poorly with subjective visual quality. PSNR has always been criticized its poor correlation with human subjective evaluations. However according to our observations, PSNR sometimes still can work very well on some specific distortion types, such as additive and quantization noise. The structural similarity (SSIM) index is a method for measuring the similarity between two images. SSIM attempts to measure the change in luminance, contrast, and structure in an image [2-4]. The multi-scale extension of SSIM, called MS-SSIM [5], produces better results than its single-scale counterpart. Recent studies conducted in [6] and [7] have demonstrated that SSIM, MS-SSIM, and VIF could offer statistically much better performance in predicting images' fidelity than the other IQA metrics. However, SSIM and MSSSIM share a common deficiency that when pooling a single quality score from the local quality map (or the local distortion measurement map), all

positions are considered to have the same importance. Feature similarity indexing maintains IQA (image quality assurance) based on the fact that human visual system (HVS) understands an image mainly according to its low-level features. The main feature of FSIM is phase congruency which is a dimensionless measure of a local structure. Actually, PC has already been used for IQA in the literature. In [8], Liu and Laganière proposed a PC-based IQA metric. In their method, PC maps are partitioned into sub-blocks of size $5 \times 5$. Then, the crosscorrelation is used to measure the similarity between two corresponding PC sub-blocks. The overall similarity score is obtained by averaging the cross correlation values from all block pairs. In [9], PC was extended to phase coherence which can be used to characterize the image blur. Based on [8], Hassen et al. proposed an NR IQA metric to assess the sharpness of an input image [10].Due to phase congruency the contrast of the image will affect Human visual system but the secondary feature of FSIM which is gradient magnitude control perception of image quality. Phase congruency and Gradient Magnitude play complementary roles in characterizing the image local quality and derive a single quality score.

\section{DESIGN METHODOLOGY}

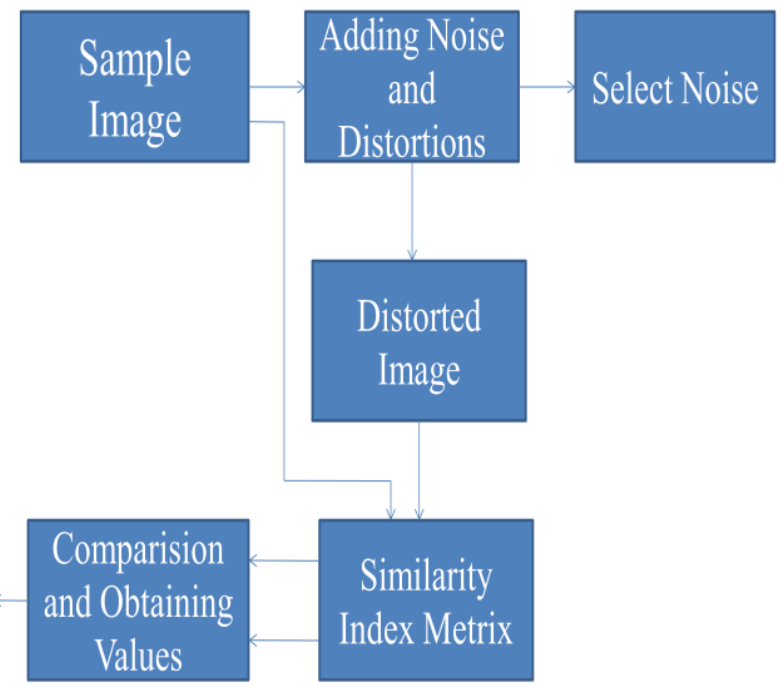

Fig. 1: Block diagram of proposed method

\section{FEATURE SIMILARITY INDEXING}

In this work, a full reference IQA is proposed based on the fact that human visual system (HVS) understands an image mainly according to its low-level features. The primary feature for this is the phase congruency (PC), which is a 
dimensionless measure of the significance of a local structure, the image gradient magnitude (GM) is employed as the secondary feature . PC and GM play complementary roles in characterizing the image local quality and derive a single quality score.

\subsection{Phase Congruency (PC)}

Phase congruency is a new method for detecting features in images. One of its significant strengths is its invariance to lighting variation within an image, as well as being able to detect a wide range of interesting features. We present a method for estimating the phase congruency of localized frequencies that cannot be measured separately by Gabor filters. We show that by measuring the ratio of the standard deviation to the mean energy between different phase shifted Gabor filters that we are able to estimate whether the localised frequencies are phase congruent. Phase congruency reflects the behaviour of the image in the frequency domain. PC is contrast invariant while the contrast information does affect HVS' perception of image quality. We describes a new corner and edge detector [12-14] developed from the phase congruency model of feature detection[11].
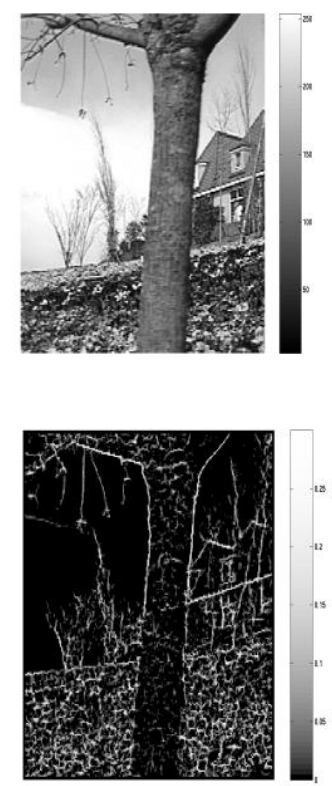

Fig. 2: (a) Original image (b) localized frequency based phase congruency
In this paper we adopt the method developed from the 1D signal I(x). Here $M_{n}^{e}$ and the $M_{n}^{O}$ are the even-symmetric and odd-symmetric filters on scale $\mathrm{n}$ and they form a quadrature pair. The signal will form a response vector at position $x$ on scale $n:\left[e_{n}(\mathrm{x}), o_{n}(\mathrm{x})\right]=\left[\mathrm{I}(\mathrm{x})^{*} M_{n}^{e}, \mathrm{I}(\mathrm{x})^{*} M_{n}^{o}\right.$ ], and the local amplitude on scale $\mathrm{n}$ is $\mathrm{A}_{\mathrm{n}}(\mathrm{x})=$ $\sqrt{e_{n}(x)^{2}+o_{n}(x)^{2}}$ Let $\mathrm{V}(\mathrm{x})=\sum_{\mathrm{n}} \mathrm{e}_{\mathrm{n}}(\mathrm{x})$ and $\mathrm{H}(\mathrm{x})=\sum_{\mathrm{n}} \mathrm{o}_{\mathrm{n}}(\mathrm{x})$

Then phase congruency is given by

$$
\mathrm{PC}(\mathrm{x})=\frac{E(x)}{\epsilon+\sum_{n} A_{n}(x)}
$$

Where $\mathrm{E}(\mathrm{x})=\sqrt{V^{2}(x)+H^{2}(x)}$

and $\varepsilon$ is a small positive constant. We adopt the log-Gabor filters because:

1) Log-Gabor filters can be constructed with arbitrary bandwidth and the bandwidth can be optimised to produce a filter with minimal spatial extent.

2) log-Gabor functions, by definition, always have no DC component.

3) The transfer function of the log-Gabor filter has an extended tail at the high frequency end, which makes it more capable to encode natural images than ordinary.

\subsection{Gradient magnitude (GM)}

Image gradient computation is a traditional topic in image processing. Gradient operators can be expressed by convolution masks. Three commonly used gradient operators are the Sobel operator, the Prewitt operator and the Scharroperator. Their performances will be examined in the section of experimental results. The partial derivatives $G_{x}(y)$ and $G_{y}(y)$ of the image $f(y)$ along horizontal and vertical directions using the three gradient operators are used. The gradient magnitude $(\mathrm{GM})$ of $\mathrm{f}(\mathrm{y})$ is then defined as $\sqrt{G_{x}^{2}+G_{y}^{2}}$

\subsection{FSIM algorithm}

With the extracted PC and GM feature maps, in this section we present a novel Feature Similarity (FSIM) index for IQA. Suppose that we are going to calculate the similarity between images $\mathrm{f}_{1}$ (test image) and $\mathrm{f}_{2}$ (original image) denote by 
$\mathrm{PC}_{1}$ and $\mathrm{PC}_{2}$. The Phase Congruency maps extracted from $\mathrm{f}_{1}(\mathrm{x})$ and $\mathrm{f}_{2}(\mathrm{x})$, and $\mathrm{G}_{1}(\mathrm{x})$ and $\mathrm{G}_{2}(\mathrm{x})$ the Gradient Magnitude maps extracted from them. It should be noted that for color images, Phase Congruency and Gradient map features are extracted from their luminance channels. FSIM will be defined and computation based on $\mathrm{PC}_{1}(\mathrm{x}), \mathrm{PC}_{2}(\mathrm{x}), \mathrm{G}_{1}(\mathrm{x})$ and $\mathrm{G}_{2}(\mathrm{x})$. Furthermore, by incorporating the image chrominance information into FSIM, an IQA index for color images or gray scale image, denoted by FSIM $_{C}$, will be obtained.

First we calculate the score of $\mathrm{PC}_{1}(\mathrm{x})$ and $\mathrm{PC}_{2}(\mathrm{x})$ and the similarity measure is defined as

$\mathrm{S}_{\mathrm{PC}}(\mathrm{x})=\frac{2 \mathrm{PC}_{1}(\mathrm{x}) \cdot \mathrm{PC}_{2}(\mathrm{x})+T_{1}}{\mathrm{PC}_{1}^{2}(\mathrm{x})+\mathrm{PC}_{2}^{2}(\mathrm{x})+\mathrm{T}_{1}}$

Where $T_{1}$ is a positive constant to increase the stability of $\mathrm{S}_{\mathrm{PC}}$. In practice, $\mathrm{T}_{1}$ can be determined based on the dynamic range of PC values. Equation shows commonly used measure to define the similarity of two positive real numbers and its result ranges within $(0,1]$.

Similarly, the $\mathrm{GM}$ values $\mathrm{G}_{1}(\mathrm{x})$ and $\mathrm{G}_{2}(\mathrm{x})$ are compared and the similarity measure is defined as

$\mathrm{S}_{\mathrm{G}}(\mathrm{x})=\frac{2 \mathrm{G}_{1}(\mathrm{x}) \cdot \mathrm{G}_{2}(\mathrm{x})+\mathrm{T}_{2}}{\mathrm{G}_{1}^{2}(\mathrm{x})+\mathrm{G}_{2}^{2}(\mathrm{x})+\mathrm{T}_{2}}$

Where $T_{2}$ is a positive constant depending on the dynamic range of GM values. In our experiments, both $T_{1}$ and $T_{2}$ will be fixed to all databases so that the proposed FSIM can be conveniently used. Then, $\mathrm{S}_{\mathrm{PC}}(\mathrm{x})$ and $\mathrm{S}_{\mathrm{G}}(\mathrm{x})$ are combined to get the similarity $S_{L}(x)$ of $f_{1}(x)$ and $\mathrm{f}_{2}(\mathrm{x})$. We define $S_{\mathrm{L}}(\mathrm{x})$ as

$\mathrm{S}_{\mathrm{L}}(\mathrm{x})=\left[\mathrm{S}_{\mathrm{PC}}(\mathrm{x})\right]^{\alpha} \cdot\left[\mathrm{S}_{\mathrm{G}}(\mathrm{x})\right]^{\beta}$

Where $\alpha$ and $\beta$ are parameters used to adjust the relative importance of PC and GM features. In this paper, we set $\alpha=\beta=1$ for simplicity.

Thus $\mathrm{S}_{\mathrm{L}}(\mathrm{x})=\left[\mathrm{S}_{\mathrm{PC}}(\mathrm{x})\right] \cdot\left[\mathrm{S}_{\mathrm{G}}(\mathrm{x})\right]$.

\section{EXPERIMENTAL RESULTS AND DISCUSSION}

\section{A. Databases and methods for comparison}

There are some publicly available image databases in the IQA community, including TID2008, CSIQ, LIVE and A57. All of them will be used here for algorithm validation and comparison. The performance of the proposed FSIM and FSIM $_{C}$ indices will be evaluated and compared with four representative IQA metrics. Four commonly used performance metrics are employed to evaluate the competing IQA metrics.

\section{B. Determination of parameters}

There are several parameters need to be determined for FSIM and $\operatorname{FSIM}_{C}$. To this end, we tuned the parameters based on a sub-data set of TID2008 database, which contains the first 8 reference images in TID2008 and the associated 544 distorted images.

\section{Gradient operator selection}

Table 1: SROCC values using three gradient operators

\begin{tabular}{|l|l|}
\hline Database & SROCC \\
\hline Sobel & 0.8797 \\
\hline Prewitt & 0.8776 \\
\hline Scharr & 0.8825 \\
\hline
\end{tabular}

In our proposed IQA metrics FSIM/FSIM ${ }_{C}$, the gradient magnitude (GM) needs to be calculated. To this end, three commonly used gradient operators were examined, and the one providing the best result was selected. Such a gradient operator selection process was carried out by assuming that all the parameters discussed earlier. The selection criterion was also that the gradient operator leading to a higher SROCC would be selected.

\section{CONCLUSION}

In this paper, we proposed a novel efficient and effective IQA index, FSIM, based on a specific visual saliency model. FSIM is designed based on the assumption that an image's visual saliency map has a close relationship with its perceptual quality. Experimental results indicate that FSIM could yield statistically better prediction performance than all the other competing methods evaluated. Thus, FSIM can be the best candidate of IQA indices for real time applications.

\section{REFERENCES}

[1].Z. Wang, A.C. Bovik, H.R. Sheikh, and E.P. Simoncelli,"Image quality assessment: from error visibility to structural similarity," IEEE Trans. IP, vol. 13, pp. 600-612, 2004. 
[2].N. Damera-Venkata, T.D. Kite, W.S. Geisler, B.L. Evans, and A.C. Bovik, "Image quality assessment based on a degradation model", IEEE Trans. Image Process., vol. 9, no. 4, pp. 636-650, Apr. 2000.

[3] D.M. Chandler and S.S. Hemami, "VSNR: a wavelet-based visual signal-to-noise ratio for natural images", IEEE Trans. Image Process., vol. 16, no. 9, pp. 2284-2298, Sep. 2007.

[4] H.R. Sheikh and A.C. Bovik, "Image information and visual quality", IEEE Trans. Image Process., vol. 15, no. 2, pp. 430-444, Feb. 2006.

[5] Z. Wang, E.P. Simoncelli, and A.C. Bovik, "Multi-scale structural similarity for image quality assessment", presented at the IEEE Asilomar Conf. Signals, Systems and Computers, Nov. 2003.

[6] H.R. Sheikh, M.F. Sabir, and A.C. Bovik, "A statistical evaluation of recent full reference image quality assessment algorithms", IEEE Trans. Image Process., vol. 15 , no. 11 , pp. 3440-3451, Nov. 2006.

[7] N. Ponomarenko, V. Lukin, A. Zelensky, K. Egiazarian, M. Carli, and F. Battisti, "TID2008 - A database for evaluation of full-reference visual quality assessment metrics", Advances of Modern Radioelectronics, vol. 10, pp.30-45, 2009.
[8] Z. Liu and R. Laganière, "Phase congruence measurement for image similarity assessment", Pattern Recognit. Letters, vol. 28, no. 1, pp. 166-172, Jan. 2007.

[9] Z. Wang and E.P. Simoncelli, "Local phase coherence and the perception of blur", in Adv. Neural Information Processing Systems., 2004, pp. 786-792.

[10] R. Hassen, Z. Wang, and M. Salama, "No reference image sharpness assessment based on local phase coherence measurement", in Proc. IEEE Int. Conf. Acoust., Speech, and Signal Processing, 2010, pp. 2434-2437

[11] P. Kovesi, "Image features from phase congruency", Videre: J. Comp. Vis. Res., vol. 1, no.3, pp. 1-26, 1999.

[12] D. Marr and E. Hildreth, "Theory of edge detection", Proc. R. Soc. Lond. B, vol. 207, no. 1167 , pp. $187-217$, Feb. 1980.

[13] M.C. Morrone and D.C. Burr, "Feature detection in human vision: a phasedependent energy model", Proc. R. Soc.Lond. B, vol. 235, no. 1280, pp. 221245, Dec. 1988.

[14] M.C. Morrone and R.A. Owens, "Feature detection from local energy", Pattern Recognit.Letters, vol. 6, no. 5, pp.303-313, Dec. 1987. 